\title{
Efficacy and safety analysis of trastuzumab and paclitaxel based regimen plus carboplatin or epirubicin as neoadjuvant therapy for clinical stage II-III, HER2-positive breast cancer patients: a phase 2, open-label, multicenter, randomized trial
}

\author{
Liang Huang ${ }^{1,2, *}$, Sheng Chen ${ }^{1,2, *}$, Wentao Yang ${ }^{2,3}$, Binghe $X u^{4}$, Tao Huang ${ }^{5}$, Hongjian \\ Yang $^{6}$, Hong Zheng ${ }^{7}$, Yongsheng Wang ${ }^{8}$, Erwei Song ${ }^{9}$, Jin Zhang ${ }^{10}$, Shude Cui ${ }^{11}$, Da \\ Pang ${ }^{12}$, Lili Tang ${ }^{13}$, Yutao Lei ${ }^{14}$, Cuizhi Geng ${ }^{15}$ and Zhiming Shao ${ }^{1,2}$ \\ ${ }^{1}$ Department of Breast Surgery, Fudan University Shanghai Cancer Center/Cancer Institute, Shanghai, China \\ 2 Department of Oncology, Shanghai Medical College, Fudan University, Shanghai, China \\ ${ }^{3}$ Department of Pathology, Cancer Center/Cancer Institute, Shanghai, China \\ ${ }^{4}$ Department of Medical Oncology, Cancer Institute and Cancer Hospital, Chinese Academy of Medical Sciences and Peking \\ Union Medical College, Beijing, China \\ ${ }^{5}$ Department of Breast and Thyroid Surgery, Union Hospital, Tongji Medical College, Huazhong University of Science and \\ Technology, Wuhan, China \\ ${ }^{6}$ Department of Breast Tumor Surgery, Zhejiang Cancer Hospital, Hangzhou, Zhejiang, China \\ 7 Department of Head and Neck and Mammary Oncology, Cancer Center and State Key Laboratory of Biotherapy, Laboratory \\ of Molecular Diagnosis of Cancer, West China Hospital, Sichuan University, Chengdu, China \\ ${ }^{8}$ Breast Cancer Center, Shandong Cancer Hospital and Institute, Jinan, China \\ ${ }^{9}$ Breast Tumor Center, Sun Yat-Sen Memorial Hospital, Sun Yat-Sen University, Guangzhou, China \\ 10 3rd Department of Breast Cancer, Tianjin Medical University Cancer Institute and Hospital, Tianjin, China \\ ${ }^{11}$ Henan Breast Cancer Center, Henan Cancer Hospital, Zhengzhou, China \\ 12 Department of Breast Surgery, The Third Affiliated Hospital of Harbin Medical University, Harbin, China \\ 13 Department of Breast Surgery, Xiangya Hospital, Central South University, Changsha, China \\ ${ }^{14}$ Department of Breast Surgery, Peking University Third Hospital, Beijing, China \\ ${ }^{15}$ Department of Breast Cancer Center, The Fourth Hospital of Hebei Medical University, Shijiazhuang, China \\ * These authors have contributed equally to this work
}

Correspondence to: Zhiming Shao, email: zhimingshao@yahoo.com

Keywords: neoadjuvant chemotherapy, trastuzumab, pathological complete response, clinical trial, carboplatin, anthracycline Received: March 23, 2015

Accepted: May 18, 2015

Published: June 02, 2015

This is an open-access article distributed under the terms of the Creative Commons Attribution License, which permits unrestricted use, distribution, and reproduction in any medium, provided the original author and source are credited.

\section{ABSTRACT}

This trial was designed to compare the efficacy and safety between epirubicin $(E)$ and carboplatin $(C)$ in combination with paclitaxel $(P)$ and trastuzumab $(H)$ in neoadjuvant setting. In 13 Chinese cancer centers, 100 patients with HER2-positive, locally advanced breast cancer were 1:1 randomized to receive medication as follows: trastuzumab and paclitaxel weekly combined with carboplatin weekly for PCH group, or epirubicin every 3 weeks for PEH group. Patients were given 4 to 6 cycles of chemotherapy. The primary endpoint was pathologic complete response (PCR) rate, which was no significant difference in $\mathrm{PCH}$ and $\mathrm{PEH}$ regimen (39.1\% vs. $48.8 \%$; $p=0.365$ ). However, PEH regimen achieved higher $P C R$ in luminal-B (HER2-poitive) subgroup ( $55.0 \%$ vs. $24.0 \% ; p=0.033)$, but not in ERBB2+ subgroup (42.9\% vs. $57.1 \% ; p=0.355)$. PEH regimen showed a favorable efficacy in PIK3CA mutated subgroup $(69.2 \%$ vs.23.5\%, $p=0.012)$. No significant difference was observed in the subgroup analysis of TP53 mutation status, PTEN expression, FCGR2A SNP and FCGR3A SNP. Both regimens as neoadjuvant chemotherapy achieve similar efficacy 


\section{and safety. PEH might improve PCR rate, especially in the luminal-B subtype and PIK3CA mutation subtype. PEH is feasible and less likely to increase the incidence of acute cardiac events compared to $\mathrm{PCH}$.}

\section{INTRODUCTION}

Neoadjuvant therapy has emerged as a successful approach to convert patients that are inoperable at diagnosis to operable, or to make breast conserving surgery possible instead of mastectomy. A pathologic complete response (pCR) after neoadjuvant therapy is a surrogate of excellent outcome of human epidermal growth hormone receptor 2 (HER2) positive breast cancer [1]. Over the past few years, a large number of clinical trials have been conducted in neoadjuvant setting, in an attempt to further refine therapeutic strategies for patients with breast cancer. Such trials have typically aimed at selecting the better regimen for each subtype, optimizing dosage of each reagent, different sequences of similar combinations of reagents, different time intervals of drug administration, and exploring the role of novel reagents.

In a pilot study, anthracycline and paclitaxel combined with trastuzumab showed promising efficacy in patients with metastatic disease, which did not cause any symptomatic cardiac dysfunction [2]. In the pretrastuzumab era, Stearns et al. showed in a small group of patients with HER2-positive tumors that the likelihood of achieving clinical complete response was higher after anthracyclines than after taxanes treatment [3]. Relatively little attention has been paid to the concurrent combination of anthracyclines and taxanes in trastuzumab-based regimens in neoadjuvant treatment. A few trials examined carboplatin in HER2-positive metastatic breast cancer or in neoadjuvant setting, but the results were controversial [4-7]. Therefore, we designed this clinical trial to compare efficacy and safety between anthracycline and carboplatin, when combined with paclitaxel and trastuzumab. Our primary objective was to compare the pCR rates between the two treatment groups. Secondary endpoints included clinical response and safety analysis. In addition, we did an explorative analysis to identify potentially targetable genetic alterations that are associated with resistance to the regimens investigated in this study.

\section{RESULTS}

\section{Baseline characteristics}

From Aug, 2011 to May, 2012, 101 patients were enrolled from 13 medical centers in China into the clinical trial. Of these patients, 1 patient did not meet inclusion criteria, 50 were randomly assigned to the $\mathrm{PCH}$ group and 50 to the PEH group (Figure 1). Baseline patients characteristics were well balanced between groups
(Table1). Three patients in PCH and 7 patients in PEH group did not complete at least 4 cycles chemotherapy because of AE, disease progression, withdrawal of consent or immediate surgery. After completing at least 4 cycles chemotherapy, 1 patient in $\mathrm{PCH}$ and 2 patients in $\mathrm{PEH}$ withdrew from this clinical trial. As predefined in the protocol, these patients were excluded from the analysis. In whole cohort, median age was 48 years (range 2965 years), 61 patients were premenopausal. 49 patients presented with clinical TNM stage II and 51 patients with stage III. 45 patients were ER positive and 37 patients were PR positive. The cut-off value of Ki67 was $14 \%$, 79 patients expressed high level of Ki67. All patients had LVEF over 55\% and the median was $66 \%$ (range $56.5 \%$ $83 \%)$.

\section{Efficacy and responses}

Over $90 \%$ of the patients experienced a clinical objective response (CR or PR) assessed by palpation, ultrasonography and/or MRI. After 2 cycles and 4 cycles, the overall clinical response did not differ between two cohorts (Table 2). During the treatment, one patient in PEH group had tumor progress. Two patients in both cohorts underwent breast-conserving surgery (BCS). 18 (39.1\%) of 46 patients in the PCH group and $20(48.8 \%)$ of 41 patients in the PEH group achieved pCR (ypT0/ is, ypN0; OR $1.4895 \% \mathrm{CI} 0.63-3.47 ; p=0.365$ ).No significant difference was noted when other definitions of pCR (ypT0/is, ypN0/+) were used. Based on Miller \& Payne grade, no significant difference in tumor regression was observed in both cohorts $(p=0.43)$. Performing subgroup analysis of patients in the $\mathrm{PCH}$ group, $13(54.2 \%)$ of 24 patients who received $5 / 6$ cycles of PCH achieved pCR, while $5(22.7 \%)$ of 22 patients who received 4 cycles had $\mathrm{pCR}(p=0.029)$. Whereas in PEH group, subgroup analysis revealed no significant difference in pCR rate in patients who were given different number of cycles of PEH ( $56.5 \%$ vs. $38.9 \%, p=0.262)$. Univariate analysis for clinical characteristics predicting pCR was performed in whole patient population (S.Table 1), revealing no significant predictor for $\mathrm{pCR}$. However, chemotherapy regimen ( $\mathrm{PCH} v s$. $\mathrm{PEH}$ ) was found to be an independent factor associated with pCR in multivariate analysis (OR: 3.606, 95\%CI: 1.153-11.274, $p=0.027$ ). Figure 2 showed the odd ratio (OR) of achieving pCR comparing PCH versus PEH with different characteristics. The results of this analysis revealed that treating patients with hormone receptor (HR) positive breast cancer with $\mathrm{PEH}$ regimen had a significantly higher chance to achieve pCR compared with $\mathrm{PCH}$ regimen (OR: $3.87,95 \% \mathrm{CI}$ : 
Table 1: Patients characteristics at baseline

\begin{tabular}{|c|c|c|c|}
\hline & $\operatorname{PCH}(\mathrm{N}=50)$ & $\operatorname{PEH}(\mathrm{N}=50)$ & $P$ value \\
\hline Median age, yr(range) & $48(29-65)$ & $47.5(30-63)$ & 0.225 \\
\hline \multicolumn{4}{|l|}{ Menopausal status } \\
\hline Premenopausal & 30 & 31 & 0.544 \\
\hline Postmenopausal & 20 & 16 & \\
\hline \multicolumn{4}{|l|}{ Clinical tumor stage } \\
\hline cT1 & 1 & 1 & 0.970 \\
\hline$\overline{\mathrm{cT} 2}$ & 32 & 34 & \\
\hline cT3 & 13 & 12 & \\
\hline cT4 & 4 & 3 & \\
\hline \multicolumn{4}{|c|}{ Clinical lymph node stage } \\
\hline $\mathrm{cN} 0$ & 7 & 8 & 0.511 \\
\hline $\mathrm{cN} 1$ & 21 & 27 & \\
\hline $\mathrm{cN} 2$ & 15 & 9 & \\
\hline $\mathrm{cN} 3$ & 6 & 6 & \\
\hline \multicolumn{4}{|l|}{ Estrogen receptor } \\
\hline Positive & 26 & 19 & 0.183 \\
\hline Negative & 23 & 29 & \\
\hline \multicolumn{4}{|l|}{ Progesterone receptor } \\
\hline Positive & 20 & 16 & 0.446 \\
\hline Negative & 29 & 32 & \\
\hline \multicolumn{4}{|l|}{ Ki67 index } \\
\hline$\geq 14 \%$ & 40 & 39 & 0.592 \\
\hline$<14 \%$ & 6 & 8 & \\
\hline \multicolumn{4}{|l|}{ LVEF } \\
\hline Median, range & $67(58-79)$ & $65(56.5-83)$ & 0.443 \\
\hline \multicolumn{4}{|l|}{ Chemotherapy cycle } \\
\hline 4 cycles & 23 & 25 & 0.382 \\
\hline Over 4 cycles & 24 & 18 & \\
\hline FCGR2A & & & 0.278 \\
\hline $\mathrm{AA}$ & 22 & 14 & \\
\hline $\mathrm{AG} / \mathrm{GG}$ & 20 & 21 & \\
\hline FCGR3A & & & 0.298 \\
\hline TT & 19 & 20 & \\
\hline $\mathrm{TG} / \mathrm{GG}$ & 23 & 15 & \\
\hline PIK3CA status & & & 0.765 \\
\hline Wide type & 25 & 22 & \\
\hline mutated & 17 & 13 & \\
\hline TP53 mutation & & & 0.868 \\
\hline Wide type & 22 & 19 & \\
\hline Mutated & 20 & 16 & \\
\hline PTEN & & & 0.693 \\
\hline Loss & 11 & 9 & \\
\hline Normal & 35 & 35 & \\
\hline
\end{tabular}


1.09-13.81, $p=0.033$ ). For patients receiving 4 cycles of neoadjuvant chemotherapy, treatment of $\mathrm{PEH}$ also significantly increased chance of achieving pCR compared with PCH treatment(OR: 4.42, 95\%CI: 1.21-16.12, $p=$ 0.021).

Safety and toxicity Most adverse events were of grades 1-2 (S. Table 2). The most frequently occurring grade $3 / 4$ adverse events were neutropenia and granulocytopenia. The incidence of febrile neutropenia was $8 \%$ in $\mathrm{PCH}$ group and $14 \%$ in $\mathrm{PEH}$ group. $\mathrm{PEH}$ regimen caused higher frequency of grade 3/4 leucopenia than $\mathrm{PCH}$ regimen $(26 \%$ vs $8 \%, p=0.017)$. Nausea, vomiting and fatigue were the most frequent nonhematological toxic effects, but these cases were mostly mild or moderate. Symptomatic congestive cardiac failure was not observed. 4 patients in $\mathrm{PCH}$ group and 5 patients in PEH group were admitted to hospital for treatmentrelated toxicities. No treatment-related death occurred.

S.Figure 2 showed LVEF at baseline, and during neoadjuvant treatment after 2 and 4 cycles. All patients in both groups maintained normal LVEF throughout the study. After 2 cycles, over 10\% reduction of LVEF was observed in 5 patients $(11.9 \%)$ in $\mathrm{PCH}$ group and 3 patients $(7.7 \%)$ in $\mathrm{PEH}$ group, while it was 3 patients $(7.3 \%)$ in $\mathrm{PCH}$ group and 1 patient $(2.7 \%)$ in $\mathrm{PEH}$ group had over $10 \%$ LVEF reduction after 4 cycles. No patient had LVEF decrease to less than $50 \%$ at any time during the study. None of the patients receiving chemotherapy developed cardiac failure and there were no chemotherapy discontinuation due to cardiac toxicity.

\section{Biomarker analysis}

PIK3CA mutation was detected In 30 (39.0\%) out of 77 tumor samples available, which were balanced between HR-positive and HR-negative tumors (46.3\% vs. $30.5 \%, p=0.156)$. The presence of PIK3CA mutation was not significantly associated with lower pCR rate. $43.3 \%$ of patients with PIK3CA-mutant tumors achieved pCR compared with $48.8 \%$ in the wild-type subgroup (OR: $0.80,95 \%$ CI: 0.316 to $2.024 ; p=0.637$ ) (Table $3)$. Based on $P I 3 K C A$ mutation status analysis, there is no statistically significant difference in efficacy to $P I K 3 C A$ wide type tumors between $\mathrm{PEH}$ and $\mathrm{PCH}$ group $(p=0.884)$. However, $\mathrm{PEH}$ regimen showed a favorable efficacy in patients with PIK3CA mutation $(69.2 \%$ vs. $23.5 \%, p=0.012$ ).

In this data set, the frequency of FCGR2A polymorphism at amino-acid 131 was $36 \mathrm{H} / \mathrm{H}(46.8 \%)$, $36 \mathrm{H} / \mathrm{R}(46.8 \%)$, and 5R/R (6.4\%). The frequency of FCGR3A polymorphism at amino-acid 158 was $39 \mathrm{~F} / \mathrm{F}$

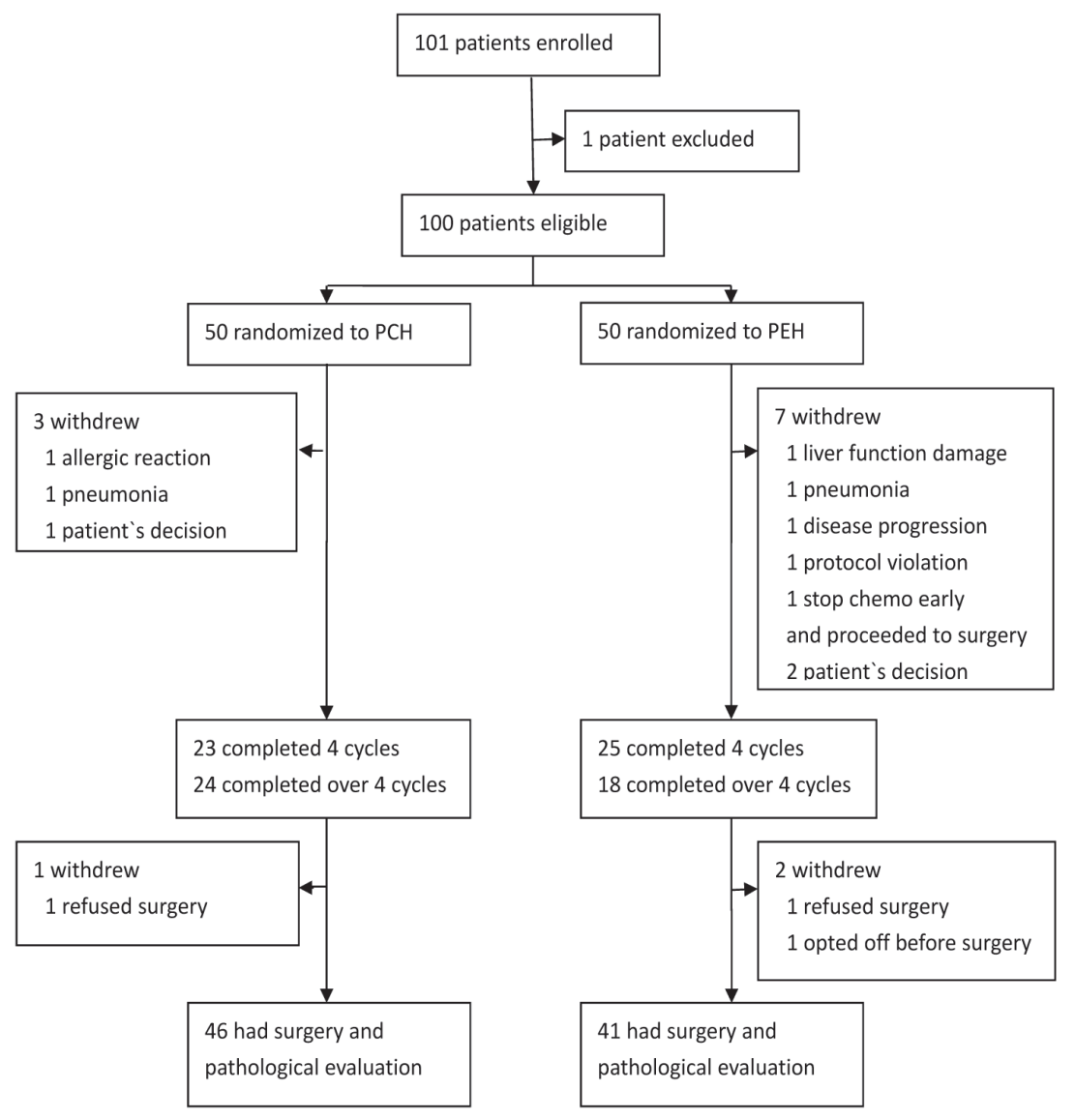

Figure 1: Flow of patients throughout the study 
Table 2: Clinical and pathological evaluation

\begin{tabular}{|c|c|c|c|c|}
\hline & РCH group & PEH group & OR $[95 \% \mathrm{CI}]$ & $P$ value \\
\hline ypT0/is,ypN0 & & & $1.481(0.632-3.472)$ & 0.365 \\
\hline No & 28 & 21 & & \\
\hline Yes & 18 & 20 & & \\
\hline ypT0/is,ypN0/+ & & & $1.645(0.704-3.847)$ & 0.249 \\
\hline No & 27 & 19 & & \\
\hline Yes & 19 & 22 & & \\
\hline MP grade for breast & & & NA & 0.431 \\
\hline MP 5 & 19 & 22 & & \\
\hline MP 4 & 11 & 8 & & \\
\hline MP 3 & 5 & 6 & & \\
\hline MP 2-0 & 11 & 5 & & \\
\hline Clinical response after 2 cycles & & & $0.651(0.171-2.474)$ & 0.740 \\
\hline $\mathrm{CR}$ & 2 & 3 & & \\
\hline PR & 40 & 40 & & \\
\hline Overall (CR or PR) & 42 & 43 & & \\
\hline SD or PD & 6 & 4 & & \\
\hline Clinical response after 4 cycles & & & $3.721(0.399-34.715)$ & 0.366 \\
\hline $\mathrm{CR}$ & 7 & 11 & & \\
\hline PR & 36 & 29 & & \\
\hline Overall (CR or PR) & 43 & 40 & & \\
\hline SD or $\mathrm{PD}$ & 4 & 1 & & \\
\hline
\end{tabular}

Subgroup

Age $\leq 45 y$

Age $>45 y$

cT1-2

cT3-4

cN Negative

cN Positive

HR Negative

HR Positive

Ki67 index $<14 \%$

Ki67 index $\geq 14 \%$

Chemotherapy 4 cycles

Chemotherapy $5 / 6$ cycles

FCGR2A H/H

FCGR2A H/R and R/R

FCGR3A F/F

FCGR3A F $N$ and V $N$

PIK3CA Wild type

PIK3CA Mutated

TP53 Wild type

TP53 Mutated

PTEN Normal

PTEN Loss

Overall

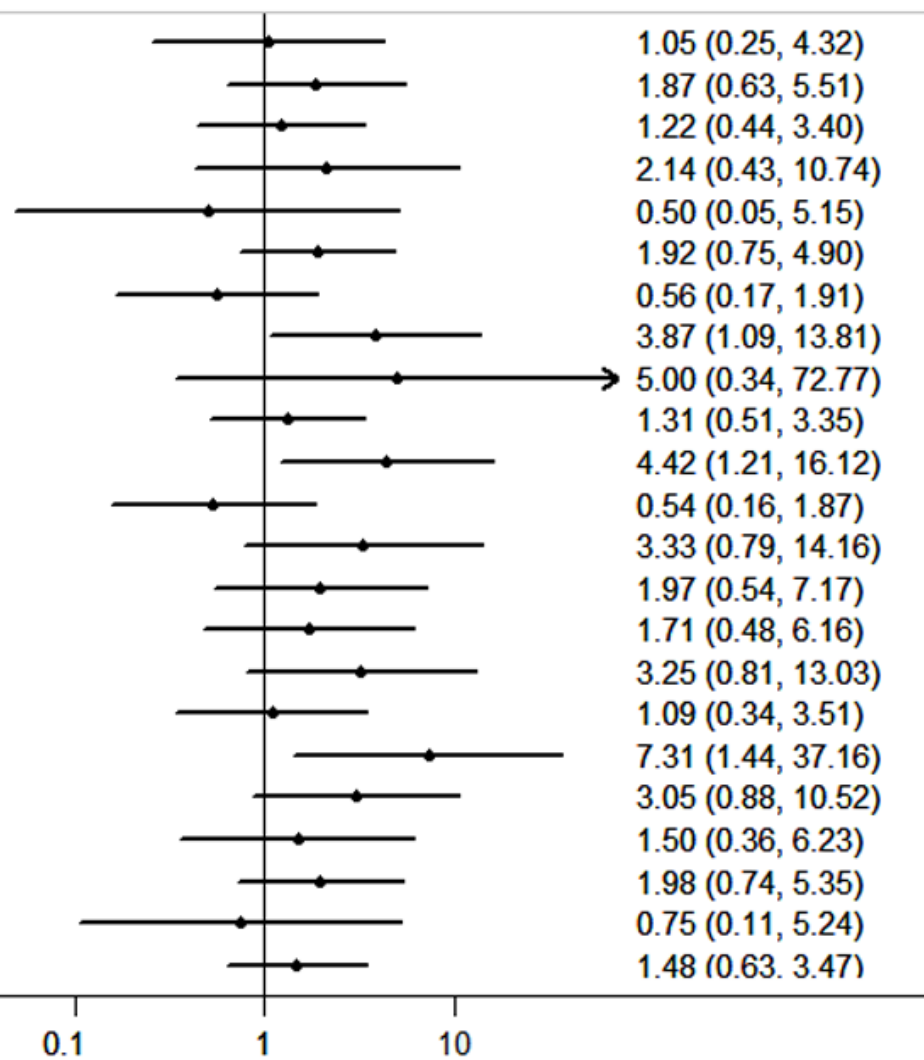

Higher pCR with PEH
OR $(95 \% \mathrm{Cl})$

$1.05(0.25,4.32)$

$1.87(0.63,5.51)$

$2.14(0.43,10.74)$

$0.50(0.05,5.15)$

$3.87(1.09,13.81)$

$(0.34,72.77)$

$4.42(1.21,16.12)$

$0.54(0.16,1.87)$

$3.33(0.79,14.16)$

$1.97(0.54,7.17)$

$1.71(0.48,6.16)$

$3.25(0.81,13.03)$

$1.09(0.34,3.51)$

$7.31(1.44,37.16)$

$3.05(0.88,10.52)$

Higher pCR with $\mathrm{PCH}$

Figure 2: Odds ratios for achieving a pCR according to subgroups 
(50.6 \%), $37 \mathrm{~F} / \mathrm{V}(48.5 \%)$, and $1 \mathrm{~V} / \mathrm{V}(1.3 \%)$. The two gene SNPs were not correlated with pCR rate. Distribution of the HER 2 mutation (A763T and T862A) was $2(2.6 \%)$, both of which did not achieved pCR. TP53 mutations in exon $4,5,6,7,8$ were detected in $32(41.6 \%)$ patients, while $20(22.2 \%)$ patients had PTEN loss. These potential biomarkers were not statistically significantly associated with $\mathrm{pCR}$ in the whole cohort and subgroup analyses.

\section{DISCUSSION}

In this clinical trial, there was no significant difference on $\mathrm{pCR}$ rate between two groups. In $\mathrm{HR}+$ subgroup and PIK3CA mutation subgroup, the $\mathrm{PEH}$ regimen could bring higher therapeutic benefit. Within the HER2-positive population, chances of achieving pCR was more common for patients with HR negative tumor than with $\mathrm{HR}$ positive tumor [8, 10, 11]. Our results showed that $\mathrm{PEH}$ improved the pCR rate in $\mathrm{HR}$ positive subtype. Few studies have evaluated concurrent administration of anthracycline-based chemotherapy and trastuzumab in neoadjuvant setting $[7,12,13]$. In contrast to previous reports, anthracycline followed by taxanes with trastuzumab-based neoadjuvant treatment was both effective and well tolerated $[14,15]$.

The biological rationale for our conclusions was based on limited preclinical and clinical observations. ER negative subtype is sensitive for chemotherapy and trastuzumab, different combinations of agents may achieve similar high pCR rate. Active agents and different regimen may improve the efficacy in luminal-B (HER2positive) subtype $[6,8,9,12]$. Results of the GeparSixto study showed an increase of the pCR rate from $37.2 \%$ to $46.7 \%$ by the addition of carboplatin. An absolute increase by $>20 \%$ was observed in patients with TNBC (37.9\% vs. 58.7\%), but no increase in patients with HER2positive breast cancer (36.3\% vs. 33.1\%) [7]. It is possible that carboplatin and docetaxel-induced senescence may render cells resistant to further therapies $[16,17]$. In a retrospective study of 300 patients, $\mathrm{PH}-\mathrm{FECH}$ (paclitaxel and trastuzumab and FEC75) shows a higher pCR rate and recurrence-free survival (RFS) advantage than TCH (docetaxel, carboplatin and trastuzumab) [13]. Previous work has shown that activation of the HER family of receptors is associated with up regulation of Topo-II $\alpha$ and increase in sensitivity to doxorubicin [18]. 30\% of HER2-potitive breast cancer express p95HER2, which is resistant to trastuzumab. However doxorubicin sensitized p95HER2 to trastuzumab in patient-derived xenografts [19]. Therefore, it is conceivable that, in HR positive and HER2 positive subgroups, which are less responsive to chemotherapy and targeted therapy, concurrently adding anthracycline may lead to additional benefit in our study.

The treatment benefits need to be weighed against the risk of cardiotoxicity. In a pivotal phase III trial in metastatic breast cancer, the concurrent administration of anthracyclines and trastuzumab resulted in an unacceptably high rate $(27 \%)$ of cardiotoxicity [20]. However, emerging evidence have showed that concurrent use of trastuzumab and anthracycline may not be associated with LVEF dysfunction. When trastuzumab was given concurrently with paclitaxel after completion of doxorubicin in adjuvant setting, the incidence of symptomatic cardiac failure was $2.8 \%-4.1 \%[21,22]$. In the monitoring of cardiac function under strict application, low cumulative dosage of anthracycline combined with trastuzumab may cause low occurrence of cardiac function failure. Despite concurrent use of doxorubicin, paclitaxel and trastuzumab in the NOAH trial, incidence of left ventricular dysfunction was less than $2 \%$. In the GeparQuattro trial, only one patient treated with concurrent administration of trastuzumab with epirubicin reported persistent decrease in LVEF to less than 50\% [23]. Additionally, the concurrent group in Z1041 trial showed that concurrent use of trastuzumab and anthracycline was not associated with an increased risk of cardiac dysfunction [12]. Consistently, no patient had LVEF decrease to less than $50 \%$ at any time during our study. Our results support the notion that trastuzumab can be given concurrently with anthracycline with low cardiac toxicity.

PIK3CA mutation in HER2-positive tumors, leading to PI3K pathway irregulation, has been shown to be important in developing resistance to trastuzumab [24]. PIK3CA mutation has been reported in approximately $20 \%$ to $30 \%$ of HER2-positive tumors by sanger sequencing of mutation hot spots [25-27]. In this study, next generation sequencing was used to detect mutations in exon4, 9, 20 of PIK3CA with detection sensitivity of $1 \%$ mutated cells. In the HER2-positive population enrolled in this study, our results showed that $\mathrm{HR}$ positive and $\mathrm{HR}$ negative tumors had similar PIK3CA mutation frequencies, which is consistent to previous pooled analysis [25]. In the neoadjuvant GepaSixto study, the presence of PIK3CA mutation was significantly associated with lower pCR rate, especially in the HR positive population. However, in the Geparquattro and GeparQuinto studies using anti-HER2 therapy, pCR rate was not significantly associated with PIK3CA mutation and HR status, which agreed with our results [25]. A retrospective study suggested that PIK3CA mutation status may be a predictor for clinical response of the combination regimen of epirubicin and docetaxel in neoadjuvant setting [28]. Comparing PCH with $\mathrm{PEH}$ in this study, our results indicated that PIK3CA mutation may be a potential predictor of clinical outcome for $\mathrm{PEH}$ regimen.

The TP53 gene is a prime candidate for predicting the response of tumors to classic chemotherapy, however other trials did not confirmed the conclusion [29]. In other studies, the TP53 mutation rate was between 20$50 \%$ in HER2 overexpressing subtype, which is similar to our results $[27,30]$. Previous retrospective studies investigating the correlation of FCGR $3 \mathrm{~A} / 2 \mathrm{~A}$ genotypes 
with clinical outcome to trastuzumab-based therapy yielded discordant results [31-33]. Our study did not show a correlation between FCGR3A-V/F and FCGR2A$\mathrm{H} / \mathrm{R}$ SNPs and pCR in patients treated with trastuzumab. While preclinical studies have shown that PTEN loss may contribute to trastuzumab resistance, previous clinical studies have failed to give us a clear answer [34-36].

From the results of this study, both PCH and PEH are suitable options for patients with locally advanced, HER2-positive breast cancer. Results of exploratory analysis implied that $\mathrm{HR}+$ and PIK3CA mutation might be a positive predictor for $\mathrm{PEH}$ treatment outcome. Our results confirmed earlier findings that concurrent administration of trastuzumab and anthracycline is not associated with an increased risk of cardiac dysfunction. However, our study may be limited by its small sample size, which is underpowered for the subgroup analysis. Although trastuzumab combined with chemotherapy remains the standard of care, dual HER2-blockade strategies have significantly improve pCR rate [8, 37]. The final survival results of the neoadjuvant trials using dual HER2 inhibition may help elucidate the final role of this approach for early stage disease. It remains to be determined whether the differences in outcomes between the groups in this trial were the result of the antitumor effect of anthracycline, or synergy from the combination of anthracyclines, paclitaxel with trastuzumab. Understanding the mechanism that underlies sensitivity and resistance to various reagents will help investigators develop rational combinations and sequences of drugs to further improve clinical outcome [38]. Future randomized studies with larger prospective cohorts and longer term follow-up are needed to validate these findings.

\section{PATIENTS AND METHODS}

\section{Study design}

This is a multicenter, randomized, phase II, parallelgroup trial conducted in 13 leading cancer centers in China. Untreated patients with histologically confirmed stage II-III, HER2-positive breast cancer were considered eligible. Other inclusion criteria included the following: age between 18-70 years; infiltrating primary breast cancer with the longest clinical diameter of more than $3.0 \mathrm{~cm}$; assessable tumor in the breast without evidence of distant metastasis measured by breast mammogram, magnetic resonance imaging, chest computed tomography scan, abdominal ultrasound and bone scan; Eastern Cooperative Oncology Group performance status of $0-1$; left ventricular ejection fraction (LVEF) $>55 \%$. Patients were required to have an adequate hematopoietic function (absolute neutrophil count $\geqq 1.5 \times 10^{9} / \mathrm{L}$, platelet count $\geqq 100 \times 10^{9} / \mathrm{L}$, and hemoglobin level $\geqq 100 \mathrm{~g} / \mathrm{L}$ ), adequate hepatic and renal function (Serum total bilirubin and Serum creatinine,$<1.5 \times \mathrm{ULN}$ (upper limit of normal), aspartate aminotransferase and alanine aminotransferase $<2.5 \times \mathrm{ULN}$ ). All patients provided written informed consent before study enrollment. The protocol was reviewed by all responsible local ethics committees. This trial is registered in ClinicalTrials.gov, with identifier NCT01428414.

\section{Procedures}

Randomization was done centrally at the operation office with sequentially numbered, opaque, sealed envelopes. The treatment plan is illustrated in S.Figure 1. Trastuzumab ( $4 \mathrm{mg} / \mathrm{kg}$ loading dose followed by 2 $\mathrm{mg} / \mathrm{kg})$ and paclitaxel $\left(75 \mathrm{mg} / \mathrm{m}^{2}\right)$ weekly combined with carboplatin (AUC $=2$ ) weekly for $\mathrm{PCH}$ group or epirubicin $\left(75 \mathrm{mg} / \mathrm{m}^{2}\right)$ every 3 weeks for PEH group. Patients were given at least 4 cycles but no more than 6 cycles under discretion of physicians, which were common accepted cycles in concurrent neoadjuvant regimens $[8,9]$. Breast surgery with axillary dissection or breast conserving surgery was performed 2-4 weeks after the last chemotherapy dose. Surgery type was decided at the surgeon's discretion. Chemotherapy regimen was at the doctors' discretion and began within 4 weeks postoperatively. One year of trastuzumab in total was recommended for all patients. Patients with estrogen or progesterone receptor-positive disease received endocrine treatment based on National Comprehensive Cancer Network (NCCN) guideline.

\section{Assessment}

Immunohistochemistry (IHC) assessment of estrogen receptor (ER), progesterone receptor (PR), HER2 expression, Ki67, PTEN was conducted in paraffinembedded tumor samples biopsied before neoadjuvant treatment according to the guidelines from the American Society of Clinical Oncology and the College of American Pathologists. HER2 positivity was determined by IHC $3+$ or fluorescence in situ hybridization (FISH) positive status. The clinical response in the breast was assessed using a breast MRI, mammogram or ultrasound after every two cycles of neoadjuvant chemotherapy and categorized as a clinical complete response, partial response, stable disease, and progressive disease according to the Response Evaluation Criteria in Solid Tumors Version 1.1. pCR in the breast was defined as the disappearance of residual invasive disease (residual ductal carcinoma in situ allowed) by pathologic examination, and pCR in the axilla was assessed as the absence of positive lymph nodes by hematoxylin and eosin staining. The independent pathological review committee included 4 senior pathologists, who did not belong to the 13 participating 
medical centers. The primary endpoint of this study was pCR in the breast and axilla. Toxicity was evaluated at every cycle and recorded according to the Common Terminology Criteria for Adverse Events (CTCAE) version 4.0.

Gene mutations were evaluated in tumor samples from FFPE core biopsies taken before therapy with a tumor content more than $50 \%$ determined by using next generation deep Ion DNA AmpliSeq ${ }^{\mathrm{TM}}$ (Average depth of coverage $=1000 \times$, Life Technologies). Hot-spot mutations were detected in whole exon $4^{\text {th }}, 9^{\text {th }}$ and $20^{\text {th }}$ of $P I 3 K C A$, exon $5^{\text {th }}-8^{\text {th }}$ exon of TP53, exon $19^{\text {th }}-25^{\text {th }}$ exon of $H E R 2$. DNA was purified from whole blood samples using QIAamp DNA Blood Mini Kit (QIAGEN, CA), and detected by PCR amplification and classical Sanger sequencing of regions containing the FCGR3A $158 \mathrm{~V} / \mathrm{F}$ and FCGR2A $131 \mathrm{H} / \mathrm{R}$ SNPs.

\section{Statistical analysis}

Patients tumor characteristics and adverse events were summarized by descriptive statistics. Treatment groups were compared by continuity corrected two-sided Pearson's $\chi 2$ test or Fisher's exact test where appropriate. The response rates and odds ratios (ORs) with 95\% confidence intervals (CIs) were calculated. Univariate logistic regression was used in subgroup analyses, while interaction effects were also displayed using forest plots. A multivariable logistic regression was used to adjust for the baseline factors. All P values were two-sided and a P value of less than 0.05 was considered statistically significant. Statistical analysis was performed using SPSS v.12.0 and STATA v.11.0.

\section{ACKNOWLEDGMENTS AND GRANT SUPPORT}

This study was supported by Shanghai Roche Pharmaceuticals Limited and China Breast Cancer Clinical Study Group.

\section{CONFLICTS OF INTEREST}

There is no conflict of interest.

\section{REFRENCES}

1. Cortazar P, Zhang L, Untch M, Mehta K, Costantino JP, Wolmark N, Bonnefoi H, Cameron D, Gianni L, Valagussa P, Swain SM, Prowell T, Loibl S, Wickerham DL, Bogaerts $\mathrm{J}$, Baselga $\mathrm{J}$, et al. Pathological complete response and longterm clinical benefit in breast cancer: the CTNeoBC pooled analysis. Lancet. 2014; 384:164-172.

2. Bianchi G, Albanell J, Eiermann W, Vitali G, Borquez D, Vigano L, Molina R, Raab G, Locatelli A, Vanhauwere B,
Gianni L and Baselga J. Pilot trial of trastuzumab starting with or after the doxorubicin component of a doxorubicin plus paclitaxel regimen for women with HER2-positive advanced breast cancer. Clin Cancer Res. 2003; 9:59445951.

3. Stearns V, Singh B, Tsangaris T, Crawford JG, Novielli A, Ellis MJ, Isaacs C, Pennanen M, Tibery C, Farhad A, Slack $\mathrm{R}$ and Hayes DF. A prospective randomized pilot study to evaluate predictors of response in serial core biopsies to single agent neoadjuvant doxorubicin or paclitaxel for patients with locally advanced breast cancer. Clin Cancer Res. 2003; 9:124-133.

4. Robert N, Leyland-Jones B, Asmar L, Belt R, Ilegbodu D, Loesch D, Raju R, Valentine E, Sayre R, Cobleigh M, Albain K, McCullough C, Fuchs L and Slamon D. Randomized phase III study of trastuzumab, paclitaxel, and carboplatin compared with trastuzumab and paclitaxel in women with HER-2-overexpressing metastatic breast cancer. J Clin Oncol. 2006; 24:2786-2792.

5. Valero V, Forbes J, Pegram MD, Pienkowski T, Eiermann W, von Minckwitz G, Roche H, Martin M, Crown J, Mackey JR, Fumoleau P, Rolski J, Mrsic-Krmpotic Z, Jagiello-Gruszfeld A, Riva A, Buyse M, et al. Multicenter phase III randomized trial comparing docetaxel and trastuzumab with docetaxel, carboplatin, and trastuzumab as first-line chemotherapy for patients with HER2-geneamplified metastatic breast cancer (BCIRG 007 study): two highly active therapeutic regimens. J Clin Oncol. 2011; 29:149-156.

6. Yu KD, Liu GY, Chen CM, Li JW, Wu J, Lu JS, Shen ZZ and Shao ZM. Weekly paclitaxel/carboplatin/trastuzumab therapy improves pathologic complete remission in aggressive HER2-positive breast cancers, especially in luminal-B subtype, compared with a once-every-3-weeks schedule. Oncologist. 2013; 18:511-517.

7. von Minckwitz G, Schneeweiss A, Loibl S, Salat C, Denkert C, Rezai M, Blohmer JU, Jackisch C, Paepke S, Gerber B, Zahm DM, Kummel S, Eidtmann H, Klare P, Huober J, Costa $\mathrm{S}$, et al. Neoadjuvant carboplatin in patients with triple-negative and HER2-positive early breast cancer (GeparSixto; GBG 66): a randomised phase 2 trial. Lancet Oncol. 2014; 15:747-756.

8. Gianni L, Pienkowski T, Im YH, Roman L, Tseng LM, Liu MC, Lluch A, Staroslawska E, de la Haba-Rodriguez J, Im SA, Pedrini JL, Poirier B, Morandi P, Semiglazov V, Srimuninnimit V, Bianchi G, et al. Efficacy and safety of neoadjuvant pertuzumab and trastuzumab in women with locally advanced, inflammatory, or early HER2-positive breast cancer (NeoSphere): a randomised multicentre, openlabel, phase 2 trial. Lancet Oncol. 2012; 13:25-32.

9. Baselga J, Bradbury I, Eidtmann H, Di Cosimo S, de Azambuja E, Aura C, Gomez H, Dinh P, Fauria K, Van Dooren V, Aktan G, Goldhirsch A, Chang TW, Horvath Z, Coccia-Portugal M, Domont J, et al. Lapatinib with trastuzumab for HER2-positive early breast cancer 
(NeoALTTO): a randomised, open-label, multicentre, phase 3 trial. Lancet. 2012; 379:633-640.

10. Gianni L, Eiermann W, Semiglazov V, Manikhas A, Lluch A, Tjulandin S, Zambetti M, Vazquez F, Byakhow M, Lichinitser M, Climent MA, Ciruelos E, Ojeda B, Mansutti M, Bozhok A, Baronio R, et al. Neoadjuvant chemotherapy with trastuzumab followed by adjuvant trastuzumab versus neoadjuvant chemotherapy alone, in patients with HER2positive locally advanced breast cancer (the NOAH trial): a randomised controlled superiority trial with a parallel HER2-negative cohort. Lancet. 2010; 375:377-384.

11. Vici P, Mottolese M, Pizzuti L, Barba M, Sperati F, Terrenato I, Di Benedetto A, Natoli C, Gamucci T, Angelucci D, Ramieri MT, Di Lauro L, Sergi D, Bartucci M, Dattilo R, Pagliuca A, et al. The Hippo transducer TAZ as a biomarker of pathological complete response in HER2positive breast cancer patients treated with trastuzumabbased neoadjuvant therapy. Oncotarget. 2014; 5:9619-9625.

12. Buzdar AU, Suman VJ, Meric-Bernstam F, Leitch AM, Ellis MJ, Boughey JC, Unzeitig G, Royce M, McCall LM, Ewer MS and Hunt KK. Fluorouracil, epirubicin, and cyclophosphamide (FEC-75) followed by paclitaxel plus trastuzumab versus paclitaxel plus trastuzumab followed by FEC-75 plus trastuzumab as neoadjuvant treatment for patients with HER2-positive breast cancer (Z1041): a randomised, controlled, phase 3 trial. Lancet Oncol. 2013; 14:1317-1325.

13. Bayraktar S, Gonzalez-Angulo AM, Lei X, Buzdar AU, Valero V, Melhem-Bertrandt A, Kuerer HM, Hortobagyi GN, Sahin AA and Meric-Bernstam F. Efficacy of neoadjuvant therapy with trastuzumab concurrent with anthracycline- and nonanthracycline-based regimens for HER2-positive breast cancer. Cancer. 2012; 118:23852393.

14. Kelly H, Kimmick G, Dees EC, Collichio F, Gatti L, Sawyer L, Ivanova A, Dressler L, Graham ML and Carey LA. Response and cardiac toxicity of trastuzumab given in conjunction with weekly paclitaxel after doxorubicin/ cyclophosphamide. Clin Breast Cancer. 2006; 7:237-243.

15. Ismael G, Hegg R, Muehlbauer S, Heinzmann D, Lum B, Kim SB, Pienkowski T, Lichinitser M, Semiglazov V, Melichar B and Jackisch C. Subcutaneous versus intravenous administration of (neo)adjuvant trastuzumab in patients with HER2-positive, clinical stage I-III breast cancer (HannaH study): a phase 3, open-label, multicentre, randomised trial. Lancet Oncol. 2012; 13:869-878.

16. Roberson RS, Kussick SJ, Vallieres E, Chen SY and Wu DY. Escape from therapy-induced accelerated cellular senescence in p53-null lung cancer cells and in human lung cancers. Cancer Res. 2005; 65:2795-2803.

17. Ewald JA, Desotelle JA, Wilding G and Jarrard DF. Therapy-induced senescence in cancer. J Natl Cancer Inst. 2010; 102:1536-1546.

18. Harris LN, Yang L, Liotcheva V, Pauli S, Iglehart JD, Colvin OM and Hsieh TS. Induction of topoisomerase
II activity after ErbB2 activation is associated with a differential response to breast cancer chemotherapy. Clin Cancer Res. 2001; 7:1497-1504.

19. Parra-Palau JL, Morancho B, Peg V, Escorihuela M, Scaltriti M, Vicario R, Zacarias-Fluck M, Pedersen K, Pandiella A, Nuciforo P, Serra V, Cortes J, Baselga J, Perou CM, Prat A, Rubio IT, et al. Effect of p95HER2/611CTF on the response to trastuzumab and chemotherapy. J Natl Cancer Inst. 2014; 106.

20. Slamon DJ, Leyland-Jones B, Shak S, Fuchs H, Paton V, Bajamonde A, Fleming T, Eiermann W, Wolter J, Pegram $\mathrm{M}$, Baselga $\mathrm{J}$ and Norton L. Use of chemotherapy plus a monoclonal antibody against HER2 for metastatic breast cancer that overexpresses HER2. N Engl J Med. 2001; 344:783-792.

21. Tan-Chiu E, Yothers G, Romond E, Geyer CE, Jr., Ewer M, Keefe D, Shannon RP, Swain SM, Brown A, Fehrenbacher L, Vogel VG, Seay TE, Rastogi P, Mamounas EP, Wolmark N and Bryant J. Assessment of cardiac dysfunction in a randomized trial comparing doxorubicin and cyclophosphamide followed by paclitaxel, with or without trastuzumab as adjuvant therapy in node-positive, human epidermal growth factor receptor 2-overexpressing breast cancer: NSABP B-31. J Clin Oncol. 2005; 23:78117819.

22. Perez EA, Suman VJ, Davidson NE, Sledge GW, Kaufman PA, Hudis CA, Martino S, Gralow JR, Dakhil SR, Ingle JN, Winer EP, Gelmon KA, Gersh BJ, Jaffe AS and Rodeheffer RJ. Cardiac safety analysis of doxorubicin and cyclophosphamide followed by paclitaxel with or without trastuzumab in the North Central Cancer Treatment Group N9831 adjuvant breast cancer trial. J Clin Oncol. 2008; 26:1231-1238.

23. Untch M, Rezai M, Loibl S, Fasching PA, Huober J, Tesch H, Bauerfeind I, Hilfrich J, Eidtmann H, Gerber B, Hanusch C, Kuhn T, du Bois A, Blohmer JU, Thomssen C, Dan Costa $\mathrm{S}$, et al. Neoadjuvant treatment with trastuzumab in HER2-positive breast cancer: results from the GeparQuattro study. J Clin Oncol. 2010; 28:2024-2031.

24. Hanker AB, Pfefferle AD, Balko JM, Kuba MG, Young CD, Sanchez V, Sutton CR, Cheng H, Perou CM, Zhao JJ, Cook RS and Arteaga CL. Mutant PIK3CA accelerates HER2-driven transgenic mammary tumors and induces resistance to combinations of anti-HER2 therapies. Proc Natl Acad Sci U S A. 2013; 110:14372-14377.

25. Loibl S, von Minckwitz G, Schneeweiss A, Paepke S, Lehmann A, Rezai M, Zahm DM, Sinn P, Khandan F, Eidtmann H, Dohnal K, Heinrichs C, Huober J, Pfitzner $\mathrm{B}$, Fasching PA, Andre F, et al. PIK3CA Mutations Are Associated With Lower Rates of Pathologic Complete Response to Anti-Human Epidermal Growth Factor Receptor 2 (HER2) Therapy in Primary HER2Overexpressing Breast Cancer. J Clin Oncol. 2014; 32:3212-3220.

26. Berns K, Horlings HM, Hennessy BT, Madiredjo M, 
Hijmans EM, Beelen K, Linn SC, Gonzalez-Angulo AM, Stemke-Hale K, Hauptmann M, Beijersbergen RL, Mills GB, van de Vijver MJ and Bernards R. A functional genetic approach identifies the PI3K pathway as a major determinant of trastuzumab resistance in breast cancer. Cancer Cell. 2007; 12:395-402.

27. Jiang YZ, Yu KD, Bao J, Peng WT and Shao ZM. Favorable prognostic impact in loss of TP53 and PIK3CA mutations after neoadjuvant chemotherapy in breast cancer. Cancer Res. 2014; 74:3399-3407.

28. Zhang Y, Liu M, Yang H, Wang J, Liu H, Li X, Li J and $\mathrm{Xu}$ J. PIK3CA mutations are a predictor of docetaxel plus epirubicin neoadjuvant chemotherapy clinical efficacy in breast cancer. Neoplasma. 2014; 61:461-467.

29. Bonnefoi H, Piccart M, Bogaerts J, Mauriac L, Fumoleau P, Brain E, Petit T, Rouanet P, Jassem J, Blot E, Zaman K, Cufer T, Lortholary A, Lidbrink E, Andre S, Litiere S, et al. TP53 status for prediction of sensitivity to taxane versus non-taxane neoadjuvant chemotherapy in breast cancer (EORTC 10994/BIG 1-00): a randomised phase 3 trial. Lancet Oncol. 2011; 12:527-539.

30. Bertheau P, Lehmann-Che J, Varna M, Dumay A, Poirot B, Porcher R, Turpin E, Plassa LF, de Roquancourt A, Bourstyn E, de Cremoux P, Janin A, Giacchetti S, Espie $\mathrm{M}$ and de The H. p53 in breast cancer subtypes and new insights into response to chemotherapy. Breast. 2013; 22 Suppl 2:S27-29.

31. Musolino A, Naldi N, Bortesi B, Pezzuolo D, Capelletti M, Missale G, Laccabue D, Zerbini A, Camisa R, Bisagni G, Neri TM and Ardizzoni A. Immunoglobulin G fragment $\mathrm{C}$ receptor polymorphisms and clinical efficacy of trastuzumab-based therapy in patients with HER-2/ neu-positive metastatic breast cancer. J Clin Oncol. 2008; 26:1789-1796.

32. Tamura K, Shimizu C, Hojo T, Akashi-Tanaka S, Kinoshita T, Yonemori K, Kouno T, Katsumata N, Ando M, Aogi K, Koizumi F, Nishio K and Fujiwara Y. FcgammaR2A and 3A polymorphisms predict clinical outcome of trastuzumab in both neoadjuvant and metastatic settings in patients with HER2-positive breast cancer. Ann Oncol. 2011; 22:13021307.

33. Hurvitz SA, Betting DJ, Stern HM, Quinaux E, Stinson J, Seshagiri S, Zhao Y, Buyse M, Mackey J, Driga A, Damaraju S, Sliwkowski MX, Robert NJ, Valero V, Crown J, Falkson C, et al. Analysis of Fcgamma receptor IIIa and IIa polymorphisms: lack of correlation with outcome in trastuzumab-treated breast cancer patients. Clin Cancer Res. 2012; 18:3478-3486.

34. Dave B, Migliaccio I, Gutierrez MC, Wu MF, Chamness GC, Wong H, Narasanna A, Chakrabarty A, Hilsenbeck SG, Huang J, Rimawi M, Schiff R, Arteaga C, Osborne CK and Chang JC. Loss of phosphatase and tensin homolog or phosphoinositol-3 kinase activation and response to trastuzumab or lapatinib in human epidermal growth factor receptor 2-overexpressing locally advanced breast cancers.
J Clin Oncol. 2011; 29:166-173.

35. Lu CH, Wyszomierski SL, Tseng LM, Sun MH, Lan KH, Neal CL, Mills GB, Hortobagyi GN, Esteva FJ and Yu D. Preclinical testing of clinically applicable strategies for overcoming trastuzumab resistance caused by PTEN deficiency. Clin Cancer Res. 2007; 13:5883-5888.

36. Barbareschi M, Cuorvo LV, Girlando S, Bragantini E, Eccher C, Leonardi E, Ferro A, Caldara A, Triolo R, Cantaloni C, Decarli N, Galligioni E and Dalla Palma P. PI3KCA mutations and/or PTEN loss in Her2-positive breast carcinomas treated with trastuzumab are not related to resistance to anti-Her2 therapy. Virchows Arch. 2012; 461:129-139.

37. Schneeweiss A, Chia S, Hickish T, Harvey V, Eniu A, Hegg R, Tausch C, Seo JH, Tsai YF, Ratnayake J, McNally $\mathrm{V}$, Ross G and Cortes J. Pertuzumab plus trastuzumab in combination with standard neoadjuvant anthracyclinecontaining and anthracycline-free chemotherapy regimens in patients with HER2-positive early breast cancer: a randomized phase II cardiac safety study (TRYPHAENA). Ann Oncol. 2013; 24:2278-2284.

38. Portier BP, Minca EC, Wang Z, Lanigan C, Gruver AM, Downs-Kelly E, Budd GT and Tubbs RR. HER4 expression status correlates with improved outcome in both neoadjuvant and adjuvant Trastuzumab treated invasive breast carcinoma. Oncotarget. 2013; 4:1662-1672. 